\title{
Modeling of macroscopic quantum states in functional properties of the laser-induced 4D- topological nanoclusters in thin films on solid surface
}

\author{
S.M. Arakelian*, A.O. Kucherik, T.A. Khudaberganov, and D.N. Bukharov \\ Vladimir State University, Department of Physics and Applied Mathematics, 600000 Vladimir, \\ Russia
}

Nanocluster structures can be easily modified in necessary direction and by controlled way in femto- nanophotonics experiments. The variation of the key topology parameters can result in new type of the quantum correlation states/size effect for charged particles.

In our earlier experiments we studied laser-induced topological nanoclusters structures of different types in thin films with unique phenomena in electrophysics and optics (see [1-3]). A simple 2-steps mechanism for enhancement of quantum behavior (e.g. in electroconductivity) exists for different conditions. First, when inelastic length $1_{\text {inelastic }}>a_{\text {cluster }}$ we have no incoherent electron-phonon $(e-p h)$ scattering, i.e. the coherent process takes place. Second, when de Broglie wave length $\lambda_{\mathrm{dB}} \equiv \ell_{\text {coh }}>\Lambda,\left(a_{\text {cluster }}-\right.$ cluster size , $\Lambda$ - spatial period of nanoparticle distribution) the coherent tunneling without loss occurs, and a long-range order with interference of the states takes place in the medium due to lattice structure.

Several results of our modeling are shown in both Fig. 1-2 for a topology structures and Fig. 3 for the Volt-Ampere characteristics.

A)
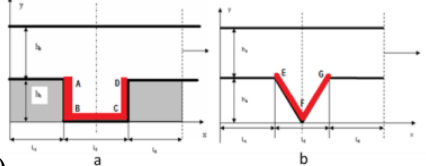

B)

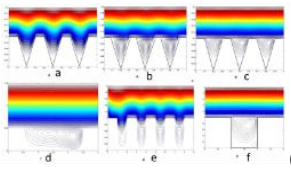

C)

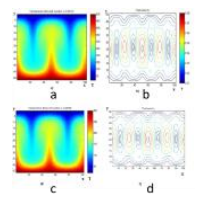

Fig.1. A) Deposition area for the channel of different shape: rectangular (a), triangular (b). B) Channel flow: with three triangular depressions at $\operatorname{Re}=10$ (a), $\operatorname{Re}=100$ (b), $\mathrm{Re}=1000$ (c); with one rectangular long shallow depression $\mathrm{Re}=1000$ (d); with four rectangular depressions $\operatorname{Re}=1000$ (e); with one rectangular $\operatorname{Re}=100000$ (f), where the Reynolds number $\operatorname{Re}=\frac{\rho U D_{r}}{\eta} ; \rho-$ medium density, $\mathrm{kg} / \mathrm{m}^{3}$; $v-$ characteristic velocity, $\mathrm{m} / \mathrm{s} ; D_{\mathrm{r}}-$ hydraulic diameter, $\mathrm{m} ; \eta-$ dynamic viscosity of the medium, $\mathrm{Pa} \cdot \mathrm{s}$ or $\mathrm{kg} /(\mathrm{m} \cdot \mathrm{s}) . \mathrm{C}) \mathrm{Graphs}$ of the temperature field (left side) and the velocity field (right side) when the lower boundary is heating up to $500{ }^{\circ} \mathrm{C}$ (a), (b); and up to $300{ }^{\circ} \mathrm{C}$ (c), (d).

\footnotetext{
*Corresponding author: arak@vlsu.ru
} 


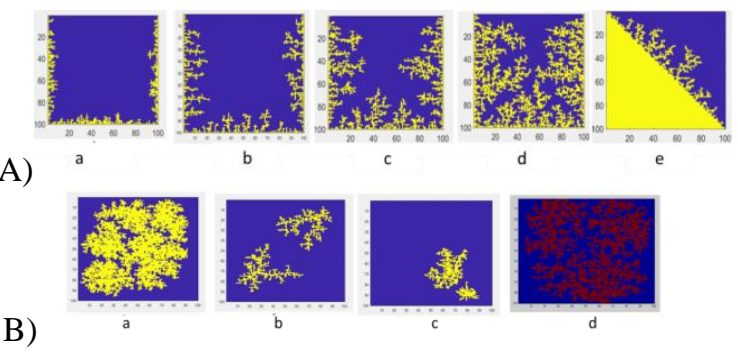

Fig. 2. A) Top panel. Aggregation centers - the line on both the side and bottom boundaries of the computational domain: (a) $\mathrm{p}=0.1, \mathrm{sc}=0.1 ;$ (b) $\mathrm{p}=0.1$, $\mathrm{sc}=0.5$; (c) $\mathrm{p}=0.5, \mathrm{sc}=1 ;$ (d) $\mathrm{p}=0.8$, $\mathrm{sc}=1 ;(\mathrm{e}) \mathrm{p}=0.5, \mathrm{sc}=1$, where: $\mathrm{sc}-$ particle sticking probability; $\mathrm{p}$-viscosity (in arbitrary units). $\mathrm{B}$ ) Low panel. Model films top view 5 islands: $\mathrm{p}=1$, $\mathrm{sc}=0.1$ (a); 2 islands: $\mathrm{p}=0.1$, $\mathrm{sc}=1$ (b); 2 islands: $\mathrm{p}=0.1, \mathrm{sc}=0.1(\mathrm{c}) ; 5$ islands: $\mathrm{p}=0.5, \mathrm{sc}=1(\mathrm{~d})$.
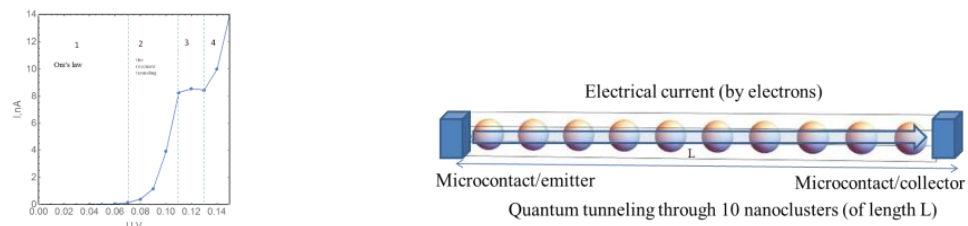

Fig. 3. Different regimes of electroconductivity in cluster systems (for Kronig-Penney model of superlattice - see 6on the right) due to relationship between electron energy and Fermi level. Vertical axis - current I, horizontal axis - voltage $\mathrm{U}$ (in arbitrary units).

In a superconductivity problem the tendency/ main trend for the phenomenon are under our study for new mechanisms of the electron coherent coupling in topological nanostructures.

The discussed phenomena give us an opportunity to establish the basis of new physical principles to create the functional elements for topological photonics in hybrid set-up (optics + electrophysics) being controlled by quantum coupled states and nonlinear dynamic processes with the necessary numerical key parameters.

\section{References}

1. Kavokin A. V., Kutrovskaya S. V., Kucherik A.O., et. al. Crossover between tunnel and hopping conductivity in granulated films of noble metals. Superlattices and Microstructures 111, 335 (2017)

2. Arakelian S.M., Kucherik A.O., Kutrovskaya S.V., et. al. Verification of quantum dimension effects in electrical conductivity with different topology of laser-induced thinfilm structures. Journal of Physics: Conference Series 951, 012018 (2018)

3. Arakelian S.M, Khudaberganov T.A., Istratov A.V., Osipov A.V., Khor'kov K.S., Topological Laser-Induced Quantum States in Nanocluster Structures: Fundamental Effects and Possible Applications (Electrical and Optical), Optics and Spectroscopy, 127, 121 (2019) 